\title{
Búsqueda de lo problémico en la enseñanza de la Física en la carrera de Técnico Superior de Biofísica Médica
} (i) (1) Q()

Search of the problemic in the teaching of Physics for medical biophysics senior technicians

Alexander Torres Hernández. ${ }^{1}$ \& Juan Jesús Mondéjar Rodríguez. ${ }^{2}$

Abstract. DOI: https://doi.org/10.33262/cienciadigital.v5i2.1596

Introduction. Problemic teaching is one of the approaches used in Cuba in order to teach Physics. Nevertheless, in the class observation of the subjects: Applied Physics and Radiation Physics for Medical Biophysics Senior Technicians in the University of Medical Sciences in Matanzas, it could be noticed that there are some limitations to find the problemic in the application of the problemic teaching by professors. Objective. to show the results of specialists' assessment on the usefulness degree of the redesigned procedure: search of problemic, for it to be applied in the lessons plan of Physics for Medical Biophysics Senior Technicians. Analysis - synthesis was the method used in the bibliography studied on didactics of Physics, problemic teaching and physics. Hypothetical - deductive method was used in the procedure redesign. A non- probabilistic sample was used, taking into account the authors' possibilities to hand in the document with the proposal to the specialists who teach physics and then, picking up the instrument. Results. the results show that the specialists' criterium about the usefulness of the didactic procedure desingned is favorable. However, an assessment of a higher number of specialists is needed, as well as the introduction of the procedure in the system of the

\footnotetext{
${ }^{1}$ Universidad de Ciencias Médicas de Matanzas, Facultad de Ciencias Médicas de Matanzas Dr. Juan Guiteras Gener, carrera de Técnico Superior en Biofísica Médica, Matanzas, Cuba, alexanderth.mtz@infomed.sld.cu, https://orcid.org/0000-0002-9235-410X

${ }^{2}$ Universidad de Matanzas, Departamento metodológico, Matanzas, Cuba, juan.mondejar@umcc.cu, https:// orcid.org/ 0000-0002-5462-9600
} 
methodological preparation of physic professors in the University of Medical Sciences of Matanzas through the development of methodological lessons and workshops. Conclusions. It is necessary to widen the study concerning the sample of specialists in order to confirm the favorable criterium of the procedure usefulness, as well as inserting it in the teaching of physics to generalize the results through the application of scientific methods, particularly in the major in Medical Biophysics Senior Technicians.

Keywords: Problemic teaching, Teaching of Physics, Didactic Procedures, medical biophysics senior technicians

\section{Resumen.}

Introducción. Uno de los enfoques utilizados en Cuba para impartir Física es la enseñanza problémica. Sin embargo, en la observación a clases de las asignaturas de Física aplicada y Física de las radiaciones en la carrera de Técnico Superior de Biofísica Médica en la Universidad de Ciencias Médicas de Matanzas, se constató que, en la aplicación de la enseñanza problémica por parte de los profesores, existen limitaciones para encontrar lo problémico. Objetivo. Mostrar los resultados de la valoración de los especialistas sobre el grado de utilidad del procedimiento rediseñado búsqueda de lo problémico, para aplicarse en la planificación de las clases de Física que se imparten en la carrera de Técnico Superior de Biofísica Médica. Metodología. Se empleó el análisis y síntesis para el estudio de la bibliografía sobre didáctica de la Física, la enseñanza problémica y física. El hipotético-deductivo para el rediseño del procedimiento. Se utilizó una muestra no probabilística, en correspondencia con las posibilidades de los autores para entregar el documento con la propuesta a los especialistas que son profesores de Física y recoger el instrumento. Resultados. Los resultados expresan que el criterio de los especialistas es favorable en cuanto a la utilidad del procedimiento didáctico diseñado. No obstante, se necesita realizar una valoración con un número superior de especialisas e introducir el procedimiento propuesto en el sistema de preparación metodológica de los profesores de Física de la Universidad de Ciencias Médicas de Matanzas mediante el desarrollo de clases metodológicas y talleres. Conclusiones. Es necesario ampliar el estudio en cuanto a la muestra de especialistas para confirmar el criterio favorable de utilidad del procedimiento diseñado e introducirlo en la enseñanza de la Física para mediante la aplicación de los métodos científicos generalizar sus resultados y en particular en la carrera de Técnico Superior de Biofísica Médica.

Palabras claves: Enseñanza problémica, Enseñanza de la Física, Procedimientos didácticos, Técnico Superior de Biofísica Médica.

\section{Introducción.}

La Física es una de las ciencias básicas que favorece la comprensión de múltiples procesos biomédicos. Su enseñanza en las carreras técnicas de la salud contribuye a formar conocimientos sólidos en los estudiantes sobre el funcionamiento biológico del cuerpo humano y de los equipos de diagnóstico y tratamiento de enfermedades. 
Uno de los enfoques utilizados en Cuba para impartir Física es la enseñanza problémica. Varios investigadores (Majmutov, 1983), (Escudero, 2016), (Escudero, González, \& García, 2016), (Gangoso, 2016) coinciden en que favorece la independencia cognoscitiva y el desarrollo de la creatividad de los estudiantes. Esta concepción de la enseñanza se emplea con mucha frecuencia en la educación médica (Pentón Velázquez, Patrón González, Hernández Pérez, \& Rodríguez, 2012). Y es muy útil para el desarrollo del proceso de enseñanza-aprendizaje de la Física en las carreras técnicas de las ciencias de la salud. Estos investigadores coinciden en la importancia de este enfoque para propiciar un aprendizaje óptimo, sin embargo, se observa la carencia de procedimientos didácticos para que los profesores determinen lo problémico en el proceso planificación de las clases, lo cual se manifiesta como una dificultad metodológica en la preparación de los docentes.

Existe consenso en (Escudero, 2016), (Escudero, González, \& Garcia, 2016), (Espinoza Freire, 2018), (Gangoso, 2016), (Pentón Velázquez, Patrón González, Hernández Pérez, \& Rodríguez, 2012), (Puig Vázquez, Curuneaux Aguilar, Chávez Jiménez, Bony del Pozo, \& Montes de Oca Selim, 2016), (Torres-Hernández \& del Toro Hechavarría, 2002), (Sardar Ali, 2019) que en la enseñanza problémica se introducen contradicciones y el profesor debe ser capaz de escogerlas y tener una actitud orientadora, dialógica, que permita a los estudiantes participar de manera activa en la búsqueda de la solución de esta, sea por la vía de la investigación o la reflexión teórica, en la que se asimilan los conocimientos y al mismo tiempo se configura como un espacio en el que se puede potenciar la creatividad. Es criterio de los autores de este texto que en ese proceso juega un rol fundamental la comunicación, no como una directiva o discurso teóricometodológico implícito, que emerge de manera espontánea, sino pensado desde la preparación metodológica del profesor cuando planifica la clase en cuestión.

Se defiende en este texto que, en este tipo de enseñanza, lo problémico es la esencia para poder diseñar actividades didácticas para su aplicación. Sin esta categoría sería imposible estructurar una situación problémica para presentarla a los estudiantes. Ella propicia y revela la condición contradictoria que provoca la necesidad de realizar una actividad para solucionar un problema.

Sin embargo, en la observación a clases de las asignaturas de Física aplicada y Física de las radiaciones en la carrera de ciclo corto de Técnico Superior de Biofísica Médica en la Universidad de Ciencias Médicas de Matanzas en Cuba, se constató que, en la aplicación de la enseñanza problémica por parte de los profesores, existen limitaciones para encontrar lo problémico, lo cual repercute en la utilidad de este método y en el aprendizaje desarrollador de los estudiantes.

Es criterio de los autores, que la didáctica de la física es deudora de procedimientos didácticos que posibiliten la búsqueda de lo problémico en el proceso de planificación de las clases. Uno de los referentes teóricos al respecto, es el procedimiento didáctico elaborado con ese propósito por (Torres-Hernández \& del Toro Hechavarría, 2002). No obstante, este necesita ser evaluado de manera teórico-práctica por los profesores de 
Física en el ejercicio de su profesión y por la comunidad científica. Para este estudio, se rediseñó y presentó a un grupo de especialistas para su valoración.

El objetivo de este artículo es mostrar los resultados de la valoración de los especialistas sobre el grado de utilidad del procedimiento rediseñado búsqueda de lo problémico, para aplicarse en la planificación de las clases de Física que se imparten en la carrera de ciclo corto de Técnico Superior de Biofísica Médica.

\section{Metodologia.}

Se empleó el análisis y síntesis para el estudio de la bibliografía sobre didáctica de la Física, la enseñanza problémica, física y la investigación de referencia presentada por (Torres-Hernández \& del Toro Hechavarría, 2002). El hipotético-deductivo para el rediseño del procedimiento. Se utilizó una muestra no probabilística, en correspondencia con las posibilidades de los autores para entregar el documento con la propuesta a los especialistas que son profesores de Física y recoger el instrumento, lo que estuvo condicionado por la situación de la pandemia de la Covid19 porque la consulta se realizó entre 3 de abril y 30 de abril de 2020. Este fue un momento en el que se implementó por el gobierno varias medidas regulatorias que impedían el desarrollo de la docencia de manera presencial y las reuniones de trabajo para evitar el contagio con el SARS-CoV2. En esta etapa se utilizó el correo electrónico y el WhatsApp como vía de intercambio de la información.

El total de especialistas consultados fue 13, cinco de la Universidad de Matanzas, cuatro de la Universidad de Ciencias Médicas de Matanzas, dos de la Universidad de Oriente y dos de la Universidad Tecnológica de la Habana. De ellos siete tienen grado científico de Doctor en Ciencias Pedagógicas, cinco tiene categoría académica de Máster en Ciencias y un licenciado. El promedio de años de experiencia es de 24,30.

Se les envió a los especialistas un documento de tres cuartillas en el que se justificaba desde los referentes teóricos y prácticos la necesidad de diseñar un procedimiento didáctico para la búsqueda de lo problémico en la implementación de la enseñanza problémica de la Física. Además, en el referido documento se encontraba el procedimiento propuesto, una encuesta con tres preguntas sobre la utilidad de la enseñanza problémica y la frecuencia con que la aplicaban, así como una tabla en la que debían marcar para cada uno de los pasos que estructuran el procedimiento propuesto en una escala valorativa sobre su utilidad.

La carrera de ciclo corto de Técnico Superior de Biofísica Médica tiene una duración de tres años y recibe la asignatura Física Aplicada en el primer semestre y Física de las radiaciones en el segundo semestre. La primera está orientada hacia la mecánica y electromagnetismo clásicos aplicados a los procesos biológicos y la segunda hacia los fundamentos nucleares que permiten emplear las radiaciones para el diagnóstico y tratamiento de diferentes patologías. 


\section{Resultados.}

Una situación problémica se encuentra relacionada con lo que pudiera ser problémico para los sujetos, en este caso para los estudiantes, de ahí la importancia de realizar un adecuado diagnóstico psicopedagógico del grupo de estudiantes, de modo que encontrar lo problémico no resulta entonces una tarea espontánea. Por esta razón la posibilidad de utilizar un procedimiento didáctico que contribuya a encontrar lo problémico en la enseñanza de la Física, resulta necesario y consecuente con los propósitos de optimización del proceso de enseñanza-aprendizaje.

Más allá de las teorizaciones sobre los procedimientos didácticos interesa aquí esclarecer que se ha entendido por estos: al conjunto de acciones didácticas - metodológicas concretas que contribuyen al logro de los objetivos parciales. Se considera que estos no son únicamente parte del método de enseñanza, también forman parte de la actividad preparatoria del docente en el proceso de planificación de las clases.

El procedimiento que se presentó a los especialistas fue el siguiente:

1. El profesor debe utilizar el diagnóstico psicopedagógico de sus estudiantes, para determinar cuáles son las habilidades intelectuales y físicas de estos, como se comporta regularmente la apropiación de los conocimientos y cuáles son los conocimientos previos que dominan. En este aspecto se incluye la cultura de la comunidad de donde proviene, así como las tradiciones, el lenguaje común y el científico.

2. Búsqueda de los hechos y fenómenos que se relacionan con el tema a tratar y son explicados por la ley o teoría que se corresponda al contenido de la clase.

3. Análisis de los hechos y fenómenos encontrados.

4. Análisis de las leyes y teorías que explican estos hechos y fenómenos.

5. Búsqueda de contradicciones entre los hechos y fenómenos con la ley y teoría que las explican.

6. Sino se encuentran, buscar contradicciones entre hechos y hechos.

7. Buscar contradicciones entre hechos y ley.

8. Buscar contradicciones entre una teoría y otra.

9. Buscar contradicciones entre hechos y teorías.

10. Buscar contradicciones entre modelo y leyes.

11. Buscar contradicciones entre modelos y hechos.

12. Buscar contradicciones entre teorías y sujeto.

13. Buscar contradicciones entre lo conocido por el sujeto (estudiante) y lo visto por él (en el caso de clase de laboratorios o experimentos).

Este importante referirse que todos estos pasos necesariamente no tienen que cumplirse, puede ser que antes de desarrollarse todos se encuentre lo problémico para una clase en particular. Sin embargo, si se realizan todos los pasos para una unidad de estudio o varias unidades, puede contribuir a obtener una visión abarcadora de lo problémico no solo en 
una clase determinada sino también en un sistema de clase, de modo que se puede señalar como un procedimiento general para este propósito.

De manera particular lo problémico puede iniciarse en una clase mediante preguntas o a través de la propia actividad que se proponga a los estudiantes durante el desarrollo de la clase y que en su ejecución se encuentre una contradicción que propicie una situación problémica.

Los resultados de la encuesta a los especialistas reveló que el $100 \%$ consideraban la enseñanza problémica como un enfoque útil para el desarrollo de la creatividad y el aprendizaje de los conocimientos físicos por parte de los estudiantes. El 69,23\% emplean la enseñanza problémica entre el $60 \%$ y el $80 \%$ de las clases. Tres profesores que representan el 23,07 \% la utilizan con poca frecuencia, entre el $30 \%$ y el $50 \%$. Y uno de ellos en menos del $30 \%$ de las clases.

Los resultados de la valoración que realizaron los especialistas sobre el grado de utilidad del procedimiento se muestran en la siguiente tabla.

Tabla 1: Respuestas sobre el grado de utilidad del procedimiento para la búsqueda de lo problémico en la enseñanza de la Física

\begin{tabular}{|c|c|c|c|c|c|}
\hline Pasos & Muy útil & Útil & $\begin{array}{c}\text { Regularmente } \\
\text { útil }\end{array}$ & Poco útil & No es útil \\
\hline 1 & 13 & & & & \\
\hline 2 & 13 & & & & \\
\hline 3 & 13 & & & & \\
\hline 4 & 10 & 3 & & & \\
\hline 5 & 12 & 1 & & & \\
\hline 6 & 13 & & & & \\
\hline 7 & 11 & 2 & & & \\
\hline 8 & 13 & & & & \\
\hline 9 & 13 & & & & \\
\hline 10 & 13 & & & & \\
\hline 11 & 13 & & & & \\
\hline 12 & 9 & 2 & 2 & & \\
\hline 13 & 13 & & & & \\
\hline
\end{tabular}

Fuente: Elaboración propia 
Estos resultados parciales expresan que el criterio de los especialistas es favorable en cuanto a la utilidad del procedimiento didáctico diseñado. No obstante, se necesita realizar una valoración con un número superior e introducir en el sistema de preparación metodológica de los profesores de Física de la Universidad de Ciencias Médicas de Matanzas mediante el desarrollo de clases metodológicas y talleres que permitan instruir para aplicarlo en la planificación de las clases de Física de las carreras técnicas de la salud.

Se considera que puede ser utilizado en cualquier enseñanza, aunque para confirmarse debe validarse mediante el método científico y su introducción en diferentes contextos educativos en los que se empleen los métodos problémicos en la enseñanza de la Física.

\section{Conclusiones.}

- Los profesores de Física Aplicada y Física de las Radiaciones de la carrera de Técnico superior de Biofísica Médica presentan limitaciones metodológicas para encontrar lo problémico cuando emplean la enseñanza problémica.

- Es necesario ampliar el estudio en cuanto a la muestra de especialistas para confirmar el criterio favorable de utilidad del procedimiento diseñado e introducirlo en la enseñanza de la Física para mediante la aplicación de los métodos científicos generalizar sus resultados y en particular en la carrera de Técnico Superior de Biofísica Médica.

\section{Bibliografía}

Cabrales Perdomo, Y., Silva Peña, J. L., \& Domínguez Reyes, A. (Abril de 2016). Procedimiento didáctico para la resolución de problemas matemáticos. Boletin Virtual, 5-4, 34-41.

Calderón Marín, C. (2018). Programa de la asignatura Física Aplicada. Ministerio de Salud Pública, La Habana.

Calderón Marín, C. F. (2018). Programa de Física de las Radiaciones. Universidad de Ciencias Médicas de la Habana, La Habana.

Escudero, C. (2016). Los procedimientos en resolución de problemas de alumnos de $3^{\circ}$ año: caracterización a través de entrevistas. Investigações em Ensino de Ciencias, $1(2), 155-175$.

Escudero, C., González, S., \& Garcia, M. (2016). Resolución de Problemas en el Aula de Física: Un análisis del discurso de su enseñanza y su aprendizaje en nivel medio. Investigaçôes em Ensino de Ciencias, 4(3), 229-251.

Espinoza Freire, E. E. (2018). Presencia de los métodos problémicos en la educación básica. Mendive, 16(2), 262-277. 
Fergusson Ramírez, E. M., Gorina Sánchez, A., Alonso Berenguer, I., \& Salgado Castillo, A. (Octubre-diciembre de 218). Perfeccionando los procedimientos didácticos para la formación investigativa de estudiantes de Ciencia de la Computación. Revista Científico Pedagógica Atenas, 4(44), 28-45.

Gangoso, Z. (2016). Investigaciones en resolución de problemas en ciencias. Investigaciones em ensino de Ciencias, 4(1), 7-50.

Gorina Sánchez, A., \& Alonso Berenguer, I. (2014). Un sistema de procedimientos didácticos para potenciar la formación del pensamiento estadístico en el nivel universitario. Órbita Pedagógica, 1(2), 41-54.

Majmutov, M. I. (1983). La enseñanza problémica. La Habana, Cuba: Pueblo y Educación.

Malaspina, U. (2016). Creación de problemas: sus potencialidades en la enseñanza y aprendizaje de las Matemáticas. Cuadernos de Investigación y Formación en Educación Matemática(15), 321-331.

Ministerio de Salud Pública. Universidad de Ciencias Médicas de la Habana. (2018). Modelo de formación profesional del nivel de educación superior de ciclo corto en Biofísica Médica. La Habana.

Pentón Velázquez, Á. R., Patrón González, A., Hernández Pérez, M. d., \& Rodríguez, Y. A. (2012). Elementos teóricos de la enseñanza problémica. Métodos y Categorías. Gaceta Médica Espirituana, 14(1).

Puig Vázquez, L., Curuneaux Aguilar, E., Chávez Jiménez, M., Bony del Pozo, P. L., \& Montes de Oca Selim, A. (2016). La enseñanza problémica como alternativa didáctica en el tema Estadísticas Sanitarias para la asignatura Metodología de la Investigación y Estadística. Información Científica, 95(6), 985-993.

Sardar Ali, S. (2019). Problem Based Learning: A Student-Centered Approach. English Language Teaching, 12(5), 73-78.

Sobrado Olalde, C. I., \& Lozano Ramos, D. (abril-junio de 2019). Propuesta de situaciones problémicas para la enseñanza y el aprendizaje de la Educación Física en la Universidad de las ciencias informáticas. OLIMPIA, 16 (55), 139-150.

Torres-Hernández, A., \& del Toro Hechavarría, Y. (2002). Procedimiento didáctico para la búsqueda de lo problémico en la enseñanza de la Física. Tesis en opción al diploma de Licenciado en Educación especialidad Física-Electrónica , Instituto Superior Pedagógico Frank País García, Física, Santiago de Cuba.

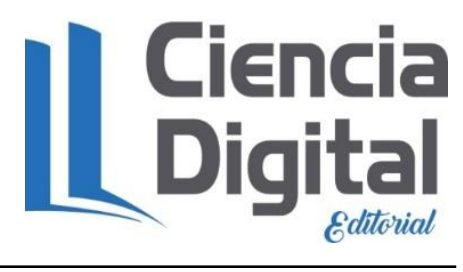




\section{PARA CITAR EL ARTÍCULO INDEXADO.}

Torres Hernández, A., \& Mondéjar Rodríguez, J. J. (2021). Búsqueda de lo problémico en la enseñanza de la Física en la carrera de Técnico Superior de Biofísica Médica . Ciencia Digital, 5(2), 100-108. https://doi.org/10.33262/cienciadigital.v5i2.1596

\section{Liencia}

El artículo que se publica es de exclusiva responsabilidad de los autores y no necesariamente reflejan el pensamiento de la Revista Ciencia Digital.

El artículo queda en propiedad de la revista y, por tanto, su publicación parcial y/o total en otro medio tiene que ser autorizado por el director de la Revista Ciencia Digital.
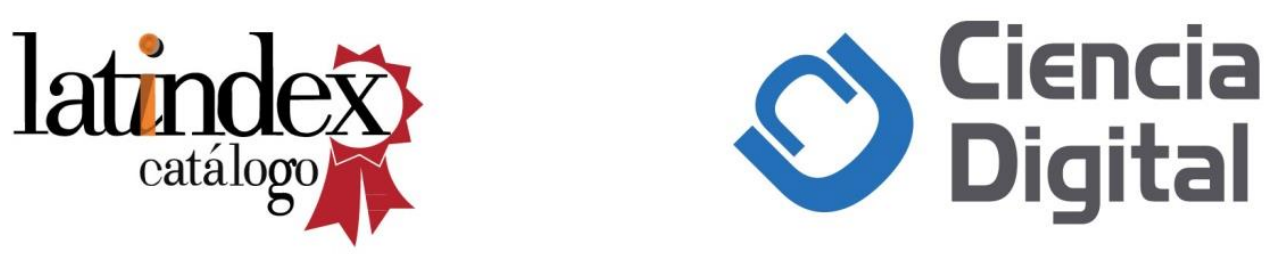